\title{
BMJ Open Effect and safety of gemtuzumab ozogamicin for the treatment of patients with acute myeloid leukaemia: a systematic review protocol
}

Nor A Muhamad (D , ,,2 Nor S Mohd Dali, ${ }^{3}$ Aliza Mohd Yacob, ${ }^{3}$ Mohd S A Kassim, ${ }^{2}$ Noor A Lodz, ${ }^{2}$ S F Abdul Wahid, ${ }^{4}$ Tahir Aris ${ }^{3}$

To cite: Muhamad NA, Mohd Dali NS, Mohd Yacob A, et al. Effect and safety of gemtuzumab ozogamicin for the treatment of patients with acute myeloid leukaemia: a systematic review protocol. BMJ Open 2020;10:e032503. doi:10.1136/ bmjopen-2019-032503

- Prepublication history and additional material for this paper are available online. To view these files, please visit the journal online (http://dx.doi org/10.1136/bmjopen-2019032503).

Received 24 June 2019 Revised 08 May 2020 Accepted 11 May 2020

Check for updates

(C) Author(s) (or their employer(s)) 2020. Re-use permitted under CC BY-NC. No commercial re-use. See rights and permissions. Published by BMJ.

${ }^{1}$ Sector for Evidence-Based, National Institutes of Health Malaysia, Shah Alam, Selangor, Malaysia

${ }^{2}$ Institute for Public Health, National Institutes of Health, Shah Alam, Selangor, Malaysia ${ }^{3}$ Institute for Medical Research, National Institutes of Health, Ministry of Health, Shah Alam, Selangor, Malaysia

${ }^{4}$ Cell Therapy Centre,

Pusat Perubatan Universiti Kebangsaan Malaysia, Cheras, Kuala Lumpur, Malaysia

Correspondence to

Dr Nor A Muhamad;

norasiahdr@gmail.com

\section{ABSTRACT}

Introduction Acute myeloid leukaemia (AML) is a type of cancer in which the bone marrow makes abnormal myeloblasts (a type of white blood cell), red blood cells or platelets. Gemtuzumab ozogamicin (G0) holds promise as a new agent that also could be efficacious in newly diagnosed AML with acceptable toxicity. This paper describes the design of a protocol to conduct a systematic review of published studies assessing G0 for the treatment of AML.

Method and analysis We will conduct a systematic review of randomised controlled trials that investigate the effect and safety of G0 for the treatment of patients with AML. We will search for any eligible articles from selected electronic databases. We will follow the Preferred Reporting Items for Systematic reviews and Meta-Analysis for study selection and reporting. We will use The Cochrane Handbook for Systematic Reviews of Interventions and Meta-Analysis as guidance to select eligible studies. All data will be extracted using a standardised data extraction form.

Ethics and dissemination There was no patient involved in this study, therefore no ethical consideration is needed. The findings of this study will be disseminated in a peer-reviewed journal and any relevant conference presentation.

PROSPERO registration number CRD42019123286.

\section{INTRODUCTION}

Acute myeloid leukaemia (AML) is a term used to represent a heterogeneous group of diseases resulting from a malignant change in the haematopoietic stem cells. In the USA, the overall incidence rate and the death rate are 3.6 and 2.8 per 100000 people per year, respectively. The incidence increases with age, with $40 \%$ of cases occurring in adults aged below 60 years and more than $50 \%$ in patients aged 60 years and above. Overall, the 5 -year survival rate for adults is $23.4 \%{ }^{1}$ Complete remission (CR) was achieved in $35 \%-40 \%$ of adult patients aged 60 years or younger and $5 \%-15 \%$ among patients older than 60 years of age. ${ }^{2}$ Mortality in patients
Strengths and limitations of this study

- We will do a wide search on all data from various databases, for example, Cochrane, PubMed, EMBASE and clinical trials.

- This study will discuss in detail about the methods for conducting a systematic review.

- The study will only include randomised controlled trials.

- The study will summarise the evidence and plan the meta-analysis for data that we can pool together.

with AML can result from treatment-related causes, relapse or primary refractoriness. The mortality rate is approximately $50 \%$ in patients aged 60 years or younger and about $80 \%$ in patients aged 60 years and above. ${ }^{34}$

Prognostic factors can be subdivided into two categories: patient-associated factors and disease-related factors. Patient-associated factors, such as advanced age, performance status and coexisting conditions, commonly predict treatment-related risks, whereas disease-related factors, such as tumour burden (white blood cell count), secondary AML (AML resulting from either antecedent haematological disorder or prior chemotherapy treatment) and genetic changes, are used to predict resistance to current standard therapy. ${ }^{56}$ Of these prognostic factors, molecular genetic lesions are additionally found to be highly predictive markers of survival. ${ }^{58}$ These markers are used in risk classification. The National Comprehensive Cancer Network defines three risk subgroups based on their cytogenetic and molecular abnormalities, namely favourable or betterrisk, intermediate-risk and poor-risk. ${ }^{49}$

The treatment for AML consists of induction, consolidation and maintenance phases. ${ }^{2} 10$ Standard induction therapy for patients aged less than 60 years most often 
consists of cytarabine (cytosine arabinoside (Ara-C)) given by continuous infusion for 7 days with an anthracycline (such as daunorubicin and idarubicin) given daily for 3 days. ${ }^{9}$ The standard of care for consolidation consists of three to four courses of high-dose intravenous Ara-C given every 12 hours on day 1,3 and $5 .{ }^{11}$ Chemotherapy is often not recommended for patients in poor health because of its toxicity. Besides antileukaemic drugs, patients would also receive supportive care such as treatment of infections (prophylactic administration of antifungal and antibacterial agent $)^{12}$ and transfusions to cover anaemia or thrombocytopenia. ${ }^{13} 14$

Gemtuzumab ozogamicin (GO) is one of the new class of monoclonal antibodies used in the treatment of AML. GO is a recombinant humanised anti-CD33 monoclonal antibody conjugated to the antitumour antibiotic, calicheamicin, which permits the drug to be targeted selectively to the CD33-positive AML blast cells, spares normal cells and thus would be expected to limit nonhaematological toxicity. ${ }^{15} \mathrm{GO}$ has been shown to be beneficial in the management of acute promyelocytic leukaemia (APL), a subtype of AML, either given as monotherapy ${ }^{16}$ or as a substitute for anthracycline therapy. ${ }^{17} 18$ This is due to the high cell surface expression of the CD33 antigen in APL blasts. In AML, a study by Castaigne et $a l^{19}$ reveals that patients treated with a single dose of GO $\left(3 \mathrm{mg} / \mathrm{m}^{3}\right)$ on day 1 of each of two consolidation cycles have significantly improved median event-free survival and overall survival (OS), but these results benefit the patients with favourable and intermediate karyotype. Besides that, a meta-analysis on seven trials involving 3942 patients revealed that the addition of GO into the induction therapy improved the relapse-free and event-free survival, but not OS. The addition of GO also increased the rate of early mortality. However, a lower cumulative dose of GO improved the OS. ${ }^{20}$ Another meta-analysis on five trials involving 1798 patients and 1798 control revealed that adding GO into the induction therapy prolonged the OS and relapse-free survival (RFS). It also decreased the resistant disease and relapse. However, GO has no effect on CR. Patients also developed risks of grade 3-4 nausea/vomiting, diarrhoea and liver aspartate transaminase elevation. ${ }^{21}$

One of the problems of standard chemotherapy is that the drug needs to be given in high doses. As the drug is delivered to both normal cells in the body and the leukaemic cells, this also causes cell death in normal cells and results in serious side effects. These side effects include hair loss, loss of appetite, mouth sores, nausea and vomiting. Therefore, high-dose chemotherapy is not suitable for the elderly. GO which is a monoclonal targeted therapy with reduced toxicity effects seems more appropriate for the treatment of elderly patients.

GO was first approved by the US Food and Drug Administration in 2000 for the treatment of older patients (age 60 years and above) with CD33-positive AML in the first relapse and who were not considered candidates for standard cytotoxic chemotherapy. ${ }^{22}$ In 2010, GO was voluntarily withdrawn from the US market due to a concern that it had failed to demonstrate any OS advantage as the treatment group had increased mortality. ${ }^{23} \mathrm{GO}$ was never officially approved for patient treatment in Europe, although it is used in clinical trials. However, the Japanese regulatory agency has sustained its approval for the usage of GO but restricted only to refractory diseases and original dosage. ${ }^{24}$ In view of this, it is necessary to systematically review the current evidence to reconsider the use of GO in the treatment of AML.

\section{Why is it important to do this review?}

Previous systematic reviews and meta-analyses revealed different results in terms of the survival analysis, dosage, and risks of GO. More analysis needs to be done in order to have a clearer view regarding the use of GO in the treatment of AML. Therefore, in this review, we will comprehensively assess the efficacy and safety of GO by including all reports on the latest clinical trials of GO.

\section{METHODS}

This review protocol followed the Preferred Reporting Items for Systematic review and Meta-Analysis (PRISMA) Protocols guidelines. ${ }^{25}$ This proposed study aims to assess the efficacy and safety of GO given either alone or in combination with cytotoxic chemotherapy regimen for the treatment of patients with newly diagnosed and relapsed AML.

\section{Patient and public involvement}

We did not involve patients or the public in our work.

\section{Criteria for considering studies for this review}

We will include all randomised controlled trials (RCTs) published in abstracts and full texts. We will exclude cross-over studies due to the carry over effect.

\section{Types of participants}

We will select adult patients of any age with newly diagnosed or relapsed AML. For studies that enrolled adults and children, we will include data for adult patients only (age 18 years and above) if the data can be separated. If this is impossible, we will include the data as presented by the authors and perform sensitivity analysis to assess the impact of excluding such studies on the overall results.

\section{Types of interventions}

We will include intervention with GO, either alone or in addition to chemotherapy with or without haematopoietic stem cell transplantation and/or other supportive therapy. We will compare with chemotherapy with or without haematopoietic stem cell transplantation or supportive care or a combination. We will accept different dosages and schedules of GO as defined by the authors of the individual studies. The chemotherapy regime employed in the intervention arm must be the same as in the control arm. 
Types of outcome measures

\section{Primary outcomes}

The primary outcome will be OS.

OS is defined as the time interval from random treatment assignment or entry into the study to death from any cause or to the last follow-up.

\section{Secondary outcomes}

1. RFS: it is defined as the time from the achievement of remission to the date of relapse or death.

2. Disease-free survival (DFS): it is defined as the period from randomisation to the first event of relapse from CR or death from any cause, whichever occurred first.

3. CR.

4. Overall remission (OR).

5. Treatment-related mortality (TRM): it is defined as the cause of death in patients with cancer that are not related to cancer, which includes infection, bleeding and organ dysfunction. It is also defined as the absence of progressive disease at death.

6. Adverse events.

7. Quality of life, measured by the number of survivors using a standardised validated questionnaire such as: The European Organization for Research and Treatment of Cancer Quality-Of-Life Questionnaire, Nottingham Health Profile and Quality Life Index QOL-E.

\section{Exclusion criteria}

We will exclude the studies that do not meet the inclusion criteria as stated earlier.

\section{Data sources and search strategy}

We will use the Cochrane highly sensitive search strategy as described in chapter 6 of the Cochrane Handbook for Systematic Reviews of Intervention. ${ }^{26}$ To reduce language bias, we will not impose any language restrictions. We will search the following databases:

1. MEDLINE (1980 to present) (online supplementary appendix 1).

2. Cochrane Central Register of Controlled Trials (CENTRAL) (The Cochrane Library, latest issue) (online supplementary appendix 2).

3. EMBASE (1980 to present) (online supplementary appendix 3).

\section{Searching other resources}

We will search conference proceedings from 2007 to present if they are not included in CENTRAL:

1. American Society of Hematology.

2. American Society of Clinical Oncology.

3. European Hematology Association.

4. European Society of Medical Oncology.

We will also search ongoing studies on the following repositories:

1. The Clinical Trials Search Portal of the World Health Organization (https://apps.who.int/trialsearch/).

2. ISRCTN Registry (http://www.isrctn.com/).

3. Hand search of the reference lists of all included studies.
Data collection and analysis

Selection of studies

We will select studies and collect data according to chapter 7 of the Cochrane Handbook for Systematic Reviews of Interventions. ${ }^{26}$ If there are multiple publications of a study, we will choose the latest publication if there are additional data provided. We will contact the authors of the included studies if we need further clarification of the data. Two authors (NAM and NSMD) will screen independently the titles and abstracts and exclude any studies that are obviously not eligible. We will resolve any disagreement through discussion or, if required, will consult a third author (SFAW). Two more authors (MSAK and NAL) will retrieve full articles from included studies and do the selection independently using an eligibility criteria form. We will refer any disagreement to a referee (TA). We will document our process of study screening and selection, including the number of identified records, included and excluded studies in a flow diagram according to PRISMA. ${ }^{25}$

\section{Data extraction and management}

Two authors (MSAK and AMY) will extract the data and independently complete the data extraction form. We will refer any disagreement to a referee (SFAW). We will independently extract data according to chapter 7 of the Cochrane Handbook for Systematic Reviews of Interventions. $^{26}$ The variables of data extraction form as described as follow:

1. General information: author, title, source, date of publication, country, language and type of study.

2. Methods: type of study, study duration, sample size, method of participant selection, clinical information, types of laboratory testing, laboratory findings and serological information if any.

3. Participants: age, gender, withdrawals and drop out, type of disease, recruitment rates, performance status, tumour burden and rate of secondary AML.

4. Types of intervention: setting, dose and duration, length of follow-up, type of treatment, type of additional or comparator chemotherapy, and supportive care.

5. Outcomes: OS, RFS, DFS, CR, OR, TRM, adverse events and quality of life.

\section{Assessment of methodological quality and reporting of data}

We will assess and report only studies meeting the inclusion criteria in a risk of bias table as suggested in chapter 8 of the Cochrane Handbook for Systematic Reviews of Interventions. ${ }^{26}$ Two authors (NAM and AMY) will independently assess the risk of bias. We will discuss any disagreement within the group, and if necessary, we will consult a third review author (TA).

We will carry out the assessment of the risk of bias based on the seven domains, namely random sequence generation, allocation concealment, blinding of participants and personnel, blinding of outcome assessment, incomplete outcome data, selective reporting and other bias. We will use the criteria in the Cochrane Collaboration's tool 
for assessing the risk of bias ${ }^{26}$ and we will judge included studies to be at high, low or unclear risk of bias in each of these domains.

\section{Measures of treatment effect}

We will express the dichotomous data as risk ratio with $95 \%$ CIs and the time-to-event outcomes as HR with 95\% CI. ${ }^{2728}$ We will express continuous data as mean difference or standardised mean difference (if the outcome is measured using different scales) and their respective 95\% CI.

\section{Dealing with missing data}

We will use the methods as suggested in chapter 16 of the Cochrane Handbook of Systematic Reviews. ${ }^{26}$ We will first contact the corresponding authors to request for any missing data. We will aim to do a complete case analysis, in which only participants with outcomes are included in the analysis. We will assess the drop-out rates of each study and use the principle of intention-to-treat analyses. We will conduct a sensitivity analysis to evaluate the impact of excluding studies with a high risk of bias under the domain of 'incomplete outcome data' in the overall results.

\section{Assessment of heterogeneity}

We will assess all the included studies with respect to their methodology as well as clinical and treatment characteristics. We will also assess other elements such as the participants, the intervention, outcomes and conduct of studies. We will visually inspect the forest plot for any evidence of heterogeneity of the treatment effect. We will evaluate the heterogeneity of treatment effects using the $\chi^{2}$ test, with a $p$ value of $<0.1$ to be considered significant. We will assess the degree of heterogeneity using the $\mathrm{I}^{2}$ statistics, with the values between $30 \%$ and $75 \%$ indicating moderate heterogeneity and more than $75 \%$ indicating considerable heterogeneity. ${ }^{26}$

\section{Assessment of reporting biases}

If we can pool at least 10 studies for a given outcome, we will create a funnel plot to assess the publication bias and use a linear regression test to analyse the degree of publication bias with a $\mathrm{p}$ value of $<0.1$ as significant for this test. ${ }^{26}$ Given that asymmetry could be caused by a relationship between effect size and sample size or by publication bias, we will examine any observed effect for clinical heterogeneity. ${ }^{29}$

\section{Data synthesis}

We will carry out analyses in Review Manager V.5.2 (Cochrane, UK) ${ }^{30}$ We will perform meta-analyses of the included studies using a fixed-effect model (eg, the generic inverse variance method for survival data outcomes and Mantel-Haenszel method for dichotomous data outcomes). We will follow the strategies according to chapter 9 of the Cochrane Handbook for Systematic Reviews of Interventions in our data management. ${ }^{26}$ We will consider studies to be sufficiently similar when they are in the same category of population, intervention, comparison and outcome and we will conduct a meta-analysis by pooling the appropriate data using Review Manager V.5. $2^{30}$ We will perform data analyses using the intention-to-treat principle, that is, using the original numbers of randomised participants allocated in the study arm as our denominator. If there are studies with distinctly different characteristics, for example, different categories of intervention, then we will separate the studies into subgroups and not provide a total pooled estimate. We will prepare a narrative synthesis for this subgroup. We will create a summary of findings (SOF) table according to the Cochrane Handbook for Systematic Reviews of interventions. ${ }^{26}$ We will choose seven major outcomes to be included in our SOF table, which will be generated using the GRADEpro software V.3.2 (GRADEpro GDT, Canada). The major outcomes to be included are as follows:

1. OS.

2. RFS.

3. DFS.

4. TRM.

5. Adverse events.

6. Quality of life.

\section{Subgroup analysis and investigation of heterogeneity}

We will assess all the included studies with respect to their methodology as well as clinical and treatment characteristics. We will also assess other elements such as the participants, the intervention, outcomes and conduct of studies. We will undertake the following subgroup analyses according to the strategies in chapter 9 of the Cochrane Handbook for Systematic Reviews of Interventions in our data analysis ${ }^{26}$ if data are available:

1. Type of population (younger, that is, age below 45 vs above 45 years or newly diagnosed vs relapsed or refractory AML).

2. Dose (monotherapy vs combination treatment).

\section{Sensitivity analysis}

We will perform sensitivity analysis to assess the impact of excluding studies with high or unclear risk of selection and attrition biases. We will perform sensitivity analysis to assess the impact on the pooled estimates if we switch to the random-effects model. ${ }^{26}$

\section{Ethics and dissemination}

There was no patient involved in this study. Hence, we did not need any ethical consideration for this study. We aim to submit our results to a peer-reviewed journal and present our findings at national and international meetings and conferences.

\section{DISCUSSION}

The usage of GO in AML treatment is still under clinical trials. The efficacy and safety of GO in the treatment of patients with AML are still not clearly described. Some studies that show that GO has increased the OS rate in favourable and intermediate-risk patients. However, some other studies also reported that GO did not affect the OS rate. Various studies on the dosage of GO in AML treatment gave a variety of results. Some countries only 
allow the usage of GO in clinical trials and some used GO concurrent with standard induction. Our study is designed to address the issue of efficacy and safety of GO in patients with AML.

This review will provide a more comprehensive answer to the usage of GO so that it will help the clinicians to assess the risk and benefits of using GO in AML treatment and also give a stepping stone for researchers to conduct more complete studies of GO. The meta-analysis of this study will provide a more thorough answer to the pharmacological strategies in the dosage of GO suitable for adult patients with AML.

Acknowledgements The authors would like to thank the Director General of Health, Malaysia for his permission to publish the findings of this study. They would also like to thank everyone who was involved, directly or indirectly, in contributing to the development of this protocol.

Contributors NAM and NSMD contributed to title registration, protocol and project plan development, and writing the protocol. AMY contributed in the development of overview method and evidence rating scheme. SFAW and MSAK contributed to developing selection of inclusion and exclusion criteria and outcome measurement and writing and developing the protocol. NAL contributed in writing protocol taxonomy for intervention and outcomes. TA contributed to project development and evidence rating scheme.

Funding This research received no specific grant from any funding agency in the public, commercial or not-for-profit sectors.

Competing interests None declared.

Patient and public involvement Patients and/or the public were not involved in the design, or conduct, or reporting, or dissemination plans of this research.

Patient consent for publication This study has been approved to publish by the Director General of Health, Ministry of Health, Malaysia (NMRR-19-145-46205).

Provenance and peer review Not commissioned; externally peer reviewed.

Open access This is an open access article distributed in accordance with the Creative Commons Attribution Non Commercial (CC BY-NC 4.0) license, which permits others to distribute, remix, adapt, build upon this work non-commercially, and license their derivative works on different terms, provided the original work is properly cited, appropriate credit is given, any changes made indicated, and the use is non-commercial. See: http://creativecommons.org/licenses/by-nc/4.0/.

ORCID iD

Nor A Muhamad http://orcid.org/0000-0002-7772-2103

\section{REFERENCES}

1 SEER. Hematopoietic and lymphoid neoplasm database, 2012. https://seer.cancer.gov/seertools/hemelymph/

2 Döhner H, Estey EH, Amadori S, et al. Diagnosis and management of acute myeloid leukemia in adults: recommendations from an international expert panel, on behalf of the European LeukemiaNet. Blood 2010;115:453-74.

3 Wouters BJ, Delwel R. Epigenetics and approaches to targeted epigenetic therapy in acute myeloid leukemia. Blood 2016;127:42-52.

4 Saultz JN, Garzon R. Acute myeloid leukemia: a concise review. J Clin Med 2016;17:33.

5 Döhner $\mathrm{H}$, Weisdorf DJ, Bloomfield CD. Acute myeloid leukemia. N Engl J Med 2015;373:1136-52.
6 Wheatley K, Brookes CL, Howman AJ, et al. Prognostic factor analysis of the survival of elderly patients with AML in the MRC AML11 and LRF AML14 trials. Br J Haematol 2009;145:598-605.

7 Smith ML, Hills RK, Grimwade D. Independent prognostic variables in acute myeloid leukaemia. Blood Rev 2011;25:39-51.

8 Grimwade D, Hills RK, Moorman AV, et al. Refinement of cytogenetic classification in acute myeloid leukemia: determination of prognostic significance of rare recurring chromosomal abnormalities among 5876 younger adult patients treated in the United Kingdom medical research council trials. Blood 2010;116:354-65.

9 O'Donnell MR, Abboud CN, Altman J, et al. Acute myeloid leukemia. J Natl Compr Canc Netw 2012;10:984-1021.

10 Khwaja A, Bjorkholm M, Gale RE, et al. Acute myeloid leukaemia. Nat Rev Dis Primers 2016;2:16010.

11 Burnett AK. Treatment of acute myeloid leukemia: are we making progress? Hematology 2012;2012:1-6.

12 Cornely OA, Maertens J, Winston DJ, et al. Posaconazole vs. fluconazole or itraconazole prophylaxis in patients with neutropenia. N Engl J Med 2007;356:348-59.

13 Rolston KVI. Neutropenic fever and sepsis: evaluation and management. In: Stosor V, Zembower TR, eds. Infectious complications in cancer patients. . Springer International Publishing, 2014: vol 161. 181-202.

14 Fey MF, Dreyling M, ESMO Guidelines Working Group. Acute myeloblastic leukaemias and myelodysplastic syndromes in adult patients: ESMO clinical practice guidelines for diagnosis, treatment and follow-up. Ann Oncol 2010;21 Suppl 5:v158-61.

15 Thol F, Schlenk RF. Gemtuzumab ozogamicin in acute myeloid leukemia revisited. Expert Opin Biol Ther 2014;14:1185-95

16 Lo-Coco F, Cimino G, Breccia M, et al. Gemtuzumab ozogamicin (mylotarg) as a single agent for molecularly relapsed acute promyelocytic leukemia. Blood 2004;104:1995-9.

17 Ravandi F, Estey E, Jones D, et al. Effective treatment of acute promyelocytic leukemia with all-trans-retinoic acid, arsenic trioxide, and gemtuzumab ozogamicin. J Clin Oncol 2009;27:504-10.

18 Estey EH, Giles FJ, Beran M, et al. Experience with gemtuzumab ozogamycin ("mylotarg") and all-trans retinoic acid in untreated acute promyelocytic leukemia. Blood 2002;99:4222-4.

19 Castaigne S, Pautas C, Terré C, et al. Effect of gemtuzumab ozogamicin on survival of adult patients with de-novo acute myeloid leukaemia (ALFA-0701): a randomised, open-label, phase 3 study. Lancet 2012;379:1508-16.

20 Kharfan-Dabaja MA, Hamadani M, Reljic T, et al. Gemtuzumab ozogamicin for treatment of newly diagnosed acute myeloid leukaemia: a systematic review and meta-analysis. $\mathrm{Br} \mathrm{J}$ Haematol 2013;163:315-25.

21 Li X, Xu SN, Qin DB, et al. Effect of adding gemtuzumab ozogamicin to induction chemotherapy for newly diagnosed acute myeloid leukemia: a meta-analysis of prospective randomized phase III trials. Ann Oncol 2014;25:455-61.

22 Bross PFet al. Approval summary. Clin Cancer Res 2001;7:1490.

23 Nelson R. Gemtuzumab voluntarily withdrawn from US market. 2010.

24 Tanimoto T, Tsubokura M, Mori J, et al. Differences in drug approval processes of 3 regulatory agencies: a case study of gemtuzumab ozogamicin. Invest New Drugs 2013;31:473-8.

25 Moher D, Liberati A, Tetzlaff J, et al. Preferred reporting items for systematic reviews and meta-analyses: the PRISMA statement. Ann Intern Med 2009;151:264.

26 Shuster JJ. Review: cochrane Handbook for systematic reviews for interventions, version 5.1.0. In: Julian PTHiggins, Green S, eds. , 2011: 2, 126-30.

27 Tierney JF, Stewart LA, Ghersi D, et al. Practical methods for incorporating summary time-to-event data into meta-analysis. Trials 2007;8:16.

28 Parmar MKB, Torri V, Stewart L. Extracting summary statistics to perform meta-analyses of the published literature for survival endpoints. Stat Med 1998;20:2815-34.

29 Egger M, Smith GD. Meta-Analysis bias in location and selection of studies. BMJ. In Press 1998;316:61-6.

30 The Nordic Cochrane Centre. Review manager (RevMan. The Cochrane Collaboration, 2014. 